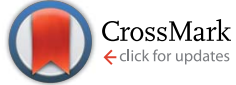

Cite this: J. Mater. Chem. A, 2016, 4, 18265

Received 9th September 2016 Accepted 2nd November 2016

DOI: $10.1039 / c 6 t a 07833 h$

www.rsc.org/MaterialsA

\section{MXene as a novel intercalation-type pseudocapacitive cathode and anode for capacitive deionization}

\author{
Pattarachai Srimuk, ${ }^{\mathrm{ab}}$ Friedrich Kaasik, ${ }^{\mathrm{ac}}$ Benjamin Krüner, ${ }^{\mathrm{ab}}$ Aura Tolosa, ${ }^{\mathrm{ab}}$ \\ Simon Fleischmann, ${ }^{b}$ Nicolas Jäckel, ${ }^{\text {ab }}$ Mehmet C. Tekeli, ${ }^{\text {ab }}$ Mesut Aslan, ${ }^{a}$ \\ Matthew E. Suss ${ }^{d}$ and Volker Presser ${ }^{\star a b}$
}

In this proof-of-concept study, we introduce and demonstrate MXene as a novel type of intercalation electrode for desalination via capacitive deionization (CDI). Traditional CDI cells employ nanoporous carbon electrodes with significant pore volume to achieve a large desalination capacity via ion electrosorption. By contrast, MXene stores charge by ion intercalation between the sheets of its two-dimensional nanolamellar structure. By this virtue, it behaves as an ideal pseudocapacitor, that is, showing capacitive electric response while intercalating both anions and cations. We synthesized $\mathrm{Ti}_{3} \mathrm{C}_{2}$-MXene by the conventional process of etching ternary titanium aluminum carbide i.e., the MAX phase $\left(\mathrm{Ti}_{3} \mathrm{AlC}_{2}\right)$ with hydrofluoric acid. The MXene material was cast directly onto the porous separator of the CDI cell without added binder, and exhibited very stable performance over 30 CDI cycles with an average salt adsorption capacity of $13 \pm 2 \mathrm{mg} \mathrm{g}^{-1}$.

\section{Introduction}

Capacitive deionization (CDI) is an emerging water treatment technology, ideally suited for the highly energy efficient removal of ions from aqueous ${ }^{1}$ or organic electrolytes. ${ }^{2}$ CDI has seen tremendous research activities with an exponentially growing number of publications over the last 5 years. Conventional CDI is based on ion removal from a feed stream by electrosorption at the electrically charged fluid/solid interface between the electrolyte and the electrode. Such CDI cells commonly utilize nanoporous carbons as electrodes, with subnanometer pores ${ }^{3}$ and/or large overall pore volumes, ${ }^{4}$ yielding the best performance values. During charging, ions are continuously removed from the feed stream until the electrode has reached the maximum ion sorption capacity for a given applied voltage. Subsequent discharging or voltage inversion regenerates the

${ }^{a} I N M$ - Leibniz Institute for New Materials, Saarbrücken, Germany. E-mail: volker. presser@leibniz-inm.de

${ }^{b}$ Department of Materials Science and Engineering, Saarland University, Saarbrücken, Germany

'IMS Laboratory, Institute of Technology, University of Tartu, Tartu, Estonia

${ }^{d}$ Faculty of Mechanical Engineering, Technion - Israel Institute of Technology, Haifa, 32000, Israel electrodes so that another CDI cycle can begin. One widely used metric to characterize CDI electrode performance is the equilibrium amount of electrosorbed salt per electrode mass (SAC $=$ salt adsorption capacity) and high values up to $c a .15-21 \mathrm{mg} \mathrm{g}^{-1}$ have been reported recently for different carbons. ${ }^{4,5}$ For example, commercial activated carbons have been found to yield up to $14 \mathrm{mg} \mathrm{g}^{-1}$, exceeded by nanotextured carbons like nitrogen doped graphene foam. ${ }^{5}$ Even higher values have been reported by introducing chemical charges to the carbon surfaces by functional groups, ${ }^{6}$ which may in the most extreme case even result in inversion of the CDI process (i.e., ion removal during discharging and ion release during charging). ${ }^{7}$ Also, in addition to the advent of novel hybrid materials, ${ }^{8-10}$ there is further progress with adapting advanced, nanotextured carbon materials for CDI. ${ }^{11,12}$

The discovery and progressing technological adaption of graphene ${ }^{13}$ has stimulated the systematic exploration of graphene analogues, that is, 2-dimensional (2-D) nanomaterials beyond carbon..$^{14}$ Recently, a novel group of 2-D nanolamellar materials has been introduced, called MXene. ${ }^{15}$ First reported in $2011,{ }^{16}$ MXenes are a fast growing group of metal carbides and nitrides that structurally originate from MAX phases. The latter are a large group of ternary transition metal carbides and nitrides with the simplifying formula $\mathrm{M}_{n+1} \mathrm{AX}_{n}$, where $n=1,2$, or 3, $\mathrm{M}$ is an early transition metal, A belongs mostly to groups 13 or 14 , and X is C and/or N. ${ }^{17}$ With weaker bonds related to Asite atoms, selective etching, for example with hydrofluoric acid $(\mathrm{HF})^{18,19}$ or electrochemical methods, ${ }^{20}$ can be achieved to transform MAX phases to MXene. Unlike graphene, MXene layers usually consist of 3,5 , or more atomic layers, depending on the MAX precursor, and the electrical and chemical properties of the exfoliated material strongly reflect the chemical process conditions. For example, MXene can be either metallically conductive, with values as high as $4000 \mathrm{~S} \mathrm{~cm}^{-1},{ }^{21}$ or semiconductor-like, ${ }^{16}$ depending on the degree of exfoliation and surface functionalization.

MXene has been reported to exhibit a large pseudocapacitance, ${ }^{22}$ outperforming the energy storage capacity of most 
other capacitive materials, especially when normalizing to the electrode volume. MXene allows for the unique ability to form binder-free electrodes (MXene paper) and to serve as an almost ideal intercalation material with rapid ion insertion between the MXene layers. In particular, MXene has exhibited high measured capacitance $\left(>300 \mathrm{~F} \mathrm{~cm}^{-3}\right)$ in lithium or sodium sulfate aqueous media, and is capable of intercalating even much larger ions, like ionic liquids or organic salts. ${ }^{2,24}$ Due to its exceptional capacitance and highly reversible intercalation/ de-intercalation of ions in aqueous media, we were motivated to explore MXene as a novel electrode material for water desalination by CDI. We here demonstrate and characterize, for the first time, a MXene CDI cell with symmetric geometry (MXene for the positively and negatively polarized electrode), which is, to the best of our knowledge, the first CDI cell with both electrodes based on an intercalation-type material.

\section{Materials and methods}

\subsection{MAX and MXene synthesis}

To obtain MXene, we first synthesized the MAX phase $\mathrm{Ti}_{3} \mathrm{AlC}_{2}$. Following the literature, ${ }^{16,25}$ this was accomplished by mixing elemental titanium (99\% purity; abcr), aluminum (99.8\% purity; Feinchemikalien und Forschungsbedarf), and carbon (type BP2000; Cabot) in a molar ratio of $3: 1.1$ : 1.88 via rigorous planetary ball milling (hard metal container, $10 \mathrm{~mm}$ hard metal balls, $20 \mathrm{~g}$ total mass in $50 \mathrm{~g}$ ethanol, $15 \mathrm{~min}$ ). The milled material was dried and cold pressed at $50 \mathrm{MPa}$ to form pellets which were heated in a graphite crucible to $1550{ }^{\circ} \mathrm{C}$ in argon atmosphere with a heating rate of $10^{\circ} \mathrm{C} \mathrm{min}{ }^{-1}$, followed by a $2 \mathrm{~h}$ heating period, and afterwards cooled to room temperature with a rate of $10^{\circ} \mathrm{C} \mathrm{min}^{-1}$.

MXene synthesis followed Naguib et al. with hydrofluoric acid (HF). ${ }^{16}$ For this, $3 \mathrm{~g}$ of MAX material was dispersed in $30 \mathrm{~mL}$ HF (40-45\%) and sealed in a polytetrafluoroethylene cup for $22 \mathrm{~h}$. The resulting material was filtered, obtaining material larger than $2 \mu \mathrm{m}$, and then centrifuged 15 times until a $\mathrm{pH}>5$ was achieved. Afterwards, the material was dried at $80{ }^{\circ} \mathrm{C}$ overnight and a yield of $2.3 \mathrm{~g}$ ( $\mathrm{ca}$. 75\%) was accomplished.

For comparison, data on commercial activated carbon from Kuraray (type YP-80F) are added and adapted from our previous work. ${ }^{26}$ YP-80F electrodes were composed of 95\% activated carbon and 5\% polytetrafluoroethylene (PTFE) polymer binder. For more information on sample preparation, see ref. 26.

\subsection{Materials characterization}

X-ray diffraction (XRD) experiments were conducted employing a D8 Advance diffractometer (Bruker AXS) with a copper X-ray source $(\mathrm{Cu} \mathrm{K \alpha}, 40 \mathrm{kV}, 40 \mathrm{~mA})$ and a nickel filter. Measurements were conducted with a 1D detector (LYNXEYE) and 2D detector (VANTAC).

Scanning electron microscope (SEM) images were recorded with a JEOL JSM 7500F field emission scanning electron microscope (JEOL, Japan) operating at $3 \mathrm{kV}$. Energy dispersive $\mathrm{X}$-ray spectroscopy (EDX) was carried out at $10 \mathrm{kV}$ in the system with an X-Max Silicon Detector from Oxford Instruments using AZtec software.
Nitrogen gas sorption measurements at $-196{ }^{\circ} \mathrm{C}$ were carried out with an Autosorb system (Autosorb 6B, Quantachrome). The samples were outgassed at $150{ }^{\circ} \mathrm{C}$ for $10 \mathrm{~h}$ under vacuum conditions at $10^{2} \mathrm{~Pa}$. The BET-specific surface area (BET-SSA) was calculated with the ASiQwin-software using the Brunauer-Emmett-Teller ${ }^{27}$ equation in the linear relative pressure range of 0.01-0.05.

\subsection{Electrode preparation and electrochemical measurements}

For full-cell experiments, ethanolic slurries containing MXene powder (250 mg powder in $100 \mathrm{~mL}$ ethanol) were prepared by ultrasound assisted stirring in ice bath for $30 \mathrm{~min}$. Porous spacer (glass fiber pre-filter, Millipore, compressed thickness of a single layer is $380 \mu \mathrm{m}$ ) was infiltrated several times by drop casting of the MXene suspension, yielding binder free coatings after drying at $120{ }^{\circ} \mathrm{C}$ for $24 \mathrm{~h}$. The exact electrode mass was determined by the mass difference of the spacer prior to and after drop casting and drying. Electrochemical measurements were conducted by symmetric full-cell cyclic voltammetry (CV; scan rate $5 \mathrm{mV} \mathrm{s}^{-1}$; up to $1.2 \mathrm{~V}$ ) and galvanostatic cycling with potential limitation to $1.2 \mathrm{~V}$ at $0.1 \mathrm{~A} \mathrm{~g}^{-1}$ in $1 \mathrm{M} \mathrm{NaCl}$ aqueous solution with a VSP300 potentiostat/galvanostat (Bio-Logic). The cell had a $\mathrm{Ag} / \mathrm{AgCl}$ spectator reference used to record the electrode potentials, while the cell voltage was not controlled by the reference (but determined as the potential difference between the two symmetric electrodes). The specific capacitance $C_{\mathrm{sp}}$ was calculated according to ref. 28 .

For half-cell experiments, $2 \mathrm{mg}$ of MXene was dispersed in $5 \mathrm{~mL}$ of ethanol. The suspension was tip-sonicated for $30 \mathrm{~min}$, coated on porous glass fiber separator and dried at $60^{\circ} \mathrm{C}$ for $6 \mathrm{~h}$. An electrode disc with a $10 \mathrm{~mm}$ diameter was cut, giving a MXene loading of $0.64 \mathrm{mg}$. As counter electrode, we used $11.4 \mathrm{mg}$ of PTFE-bound (5 mass\%) activated carbon (YP-80F) and a glass fiber (GF/A, Whatman) separator.

\subsection{Capacitive deionization experiments}

For CDI experiments, the MXene material was casted directly on the porous separator instead of casting it onto the graphite current collector or using it as a freestanding film. This approach was motivated by the need of filtration to obtain MXene paper and the most facile way of realizing this was to directly use the porous separator used for the CDI cell. To the best of our knowledge, our work is the first time that a CDI electrode was cast on the separator instead of casting onto the current collector. For this, MXene powder was dispersed in ethanol and tip-sonicated for $30 \mathrm{~min}$. Afterwards, the MXene suspension was drop casted over the glass fiber separator and dried at $60{ }^{\circ} \mathrm{C}$ for $24 \mathrm{~h}$.

We used a CDI setup described in ref. 26 with flow between electrodes (i.e., feed water flows in parallel to and in-between the electrode pair; see definition from ref. 1). The CDI stack was built from graphite current collectors (SGL Technologies; thickness: $250 \mu \mathrm{m}$ ) with attached MXene electrodes directly deposited onto the porous spacer. The CDI electrode mass was $100 \mathrm{mg}$ with a thickness of $125 \mu \mathrm{m}$, as confirmed by SEM. The 
measurements were carried out with one symmetric pair of electrodes. The total electrolyte flow rate was $22 \mathrm{~mL} \mathrm{~min}^{-1}$. Ion adsorption and desorption steps were carried out using constant potential mode at $1.2 \mathrm{~V}$ and regeneration was accomplished at $0 \mathrm{~V}$. For all electrochemical operations, we used a VSP300 potentiostat/galvanostat (Bio-Logic) and the duration of each half-cycle was $30 \mathrm{~min}$. All experiments were carried out with de-aerated $5 \mathrm{mM} \mathrm{NaCl}$ solution in a $10 \mathrm{~L}$ electrolyte tank and the electrolyte was de-aerated by flushing nitrogen gas. The salt adsorption capacity and the measured charge were defined per mass of active material (i.e., MXene mass or mass of activated carbon, for comparison) in both electrodes and were calculated as an average value from adsorption and desorption step. For quantification of the electrical charge, the leakage current measured at the end of each half-cycle was subtracted. ${ }^{29}$

The AC experimental data was taken from previous work ${ }^{26}$ with $6 \times 6 \mathrm{~cm}^{2}$ electrodes with a mass of $500 \mathrm{mg}$ per electrode pair.

\section{Results and discussion}

\subsection{Material characterization}

The obtained powder of the ternary carbide (Fig. 1A) was typical for the MAX phases with a layered morphology, which transitioned to a much more delaminated structure after HF etching (Fig. 1B). ${ }^{30}$ The drastic change in structure is also documented by X-ray powder diffraction (Fig. 1E). In particular, we see a strong shift of the (002)-peak at $9.52^{\circ} 2 \theta$ (MAX) to $8.96^{\circ} 2 \theta$ (MXene), which corresponds with an increase in $d$-space of $6.2 \%$. This shift is accompanied by an increase in the full-width at half maximum (FWHM) from $0.057^{\circ} 2 \theta$ (MAX) to $0.474^{\circ} 2 \theta$ (MXene). ${ }^{15,30}$

Chemical analysis via energy dispersive X-ray spectroscopy (EDX) shows that $\mathrm{Al}$ removal was almost fully achieved (Fig. 1D and Table 1). Small amounts of residual aluminum stem from few incompletely transformed MAX grains, which also contribute to the sharp reflections seen in the XRD pattern
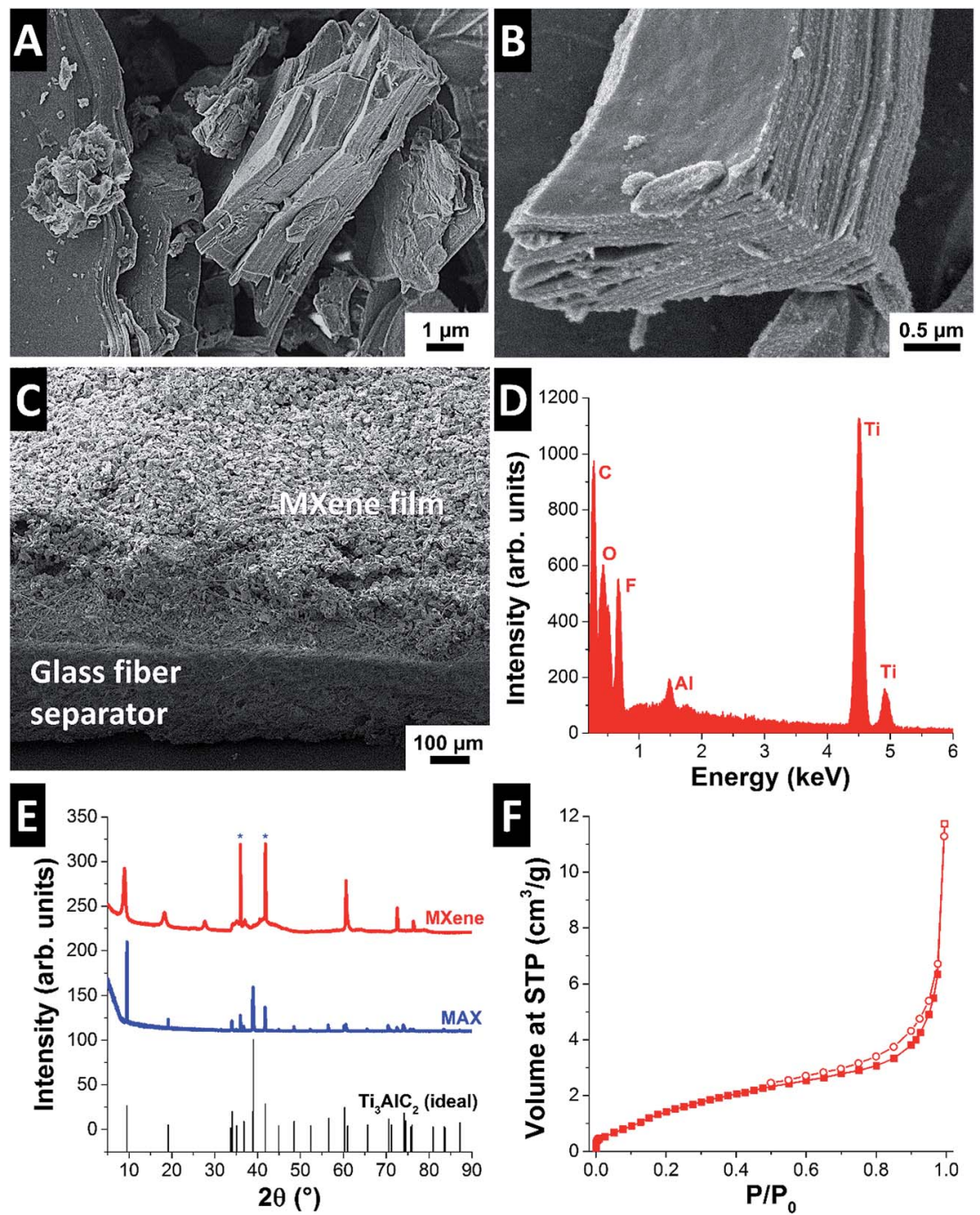

Fig. 1 Scanning electron micrographs of (A) MAX phase $\mathrm{Ti}_{3} \mathrm{AlC}_{2}$, (B) MXene $\mathrm{Ti}_{3} \mathrm{C}_{2}$, and (C) MXene $\mathrm{Ti}_{3} \mathrm{C}_{2}$ deposited onto the glass fiber separator (cross section). (D) Energy dispersive X-ray spectrum (EDX) of $M X e n e \mathrm{Ti}_{3} \mathrm{C}_{2}$. (E) X-ray diffractograms of $M A X$ and $M X$ ene phase compared to the ideal peak positions for $\mathrm{Ti}_{3} \mathrm{AlC}_{2}$ (PDF 52-0875). (F) Nitrogen gas sorption isotherm recorded at $-196{ }^{\circ} \mathrm{C}$ of $\mathrm{MXene} \mathrm{Ti}_{3} \mathrm{C}_{2}$ with a calculated $\mathrm{BET}$ surface area of $6 \mathrm{~m}^{2} \mathrm{~g}^{-1}$. 
Table 1 Elemental chemical analysis of the initial MXene material and post mortem analysis after CDI cycling

\begin{tabular}{lllllll}
\hline (Mass\%) & $\mathrm{C}$ & $\mathrm{Ti}$ & $\mathrm{O}$ & $\mathrm{Al}$ & $\mathrm{F}$ & $\mathrm{Na} \& \mathrm{Cl}$ \\
\hline Initial & $15 \pm 1$ & $59 \pm 5$ & $10 \pm 2$ & $<1$ & $14 \pm 2$ & - \\
Post mortem anode & $12 \pm 3$ & $52 \pm 8$ & $29 \pm 7$ & $<2$ & $4 \pm 1$ & $<2$ \\
Post mortem cathode & $14 \pm 4$ & $62 \pm 10$ & $14 \pm 5$ & $<1$ & $8 \pm 5$ & $<1$
\end{tabular}

(marked by stars in Fig. 1E). In addition to Ti and C, the MXene material also shows significant amounts of $\mathrm{F}$ (14 mass\%) and $\mathrm{O}$ (10 mass\%) as shown in Table 1. Etching of MAX in aqueous HF is known to yield an excess of fluorine and oxygen containing surface functional groups, where "surface" refers also the space between the MXene nanosheets. ${ }^{31}$

Although the removal of aluminum induces changes in the morphology and structure, it does not yield a large surface area. Nitrogen gas sorption analysis $-196{ }^{\circ} \mathrm{C}$ showed a BET surface area of $6 \mathrm{~m}^{2} \mathrm{~g}^{-1}$ (Fig. 1F). Typical CDI electrodes based on ion electrosorption into EDLs have BET surface areas exceeding $1000 \mathrm{~m}^{2} \mathrm{~g}^{-1} \cdot{ }^{29}$ However, as we show in this work, ion intercalation in MXene, giving rise to pseudocapacitive charge transfer and ion immobilization, allows for a high SAC without the need for a high BET surface area.

\subsection{Electrochemical behavior and capacitive deionization}

A first assessment of the ion storage capacity and electrochemical behavior of MXene in saline media was carried out by cyclic voltammetry (CV) in aqueous $1 \mathrm{M} \mathrm{NaCl}$ (Fig. 2A). As can be seen, the shape of the cyclic voltammogram is highly rectangular for activated carbon and slightly distorted for MXene. The latter features are likely due to the charging (dynamic) response of the rather thick MXene electrode. We here used roughly 100 $\mu \mathrm{m}$ thick MXene electrodes, while in the literature, usually much thinner MXene electrodes are used, such as $2 \mu \mathrm{m}$ in ref. 32. Both MXene and activated carbon are characterized by
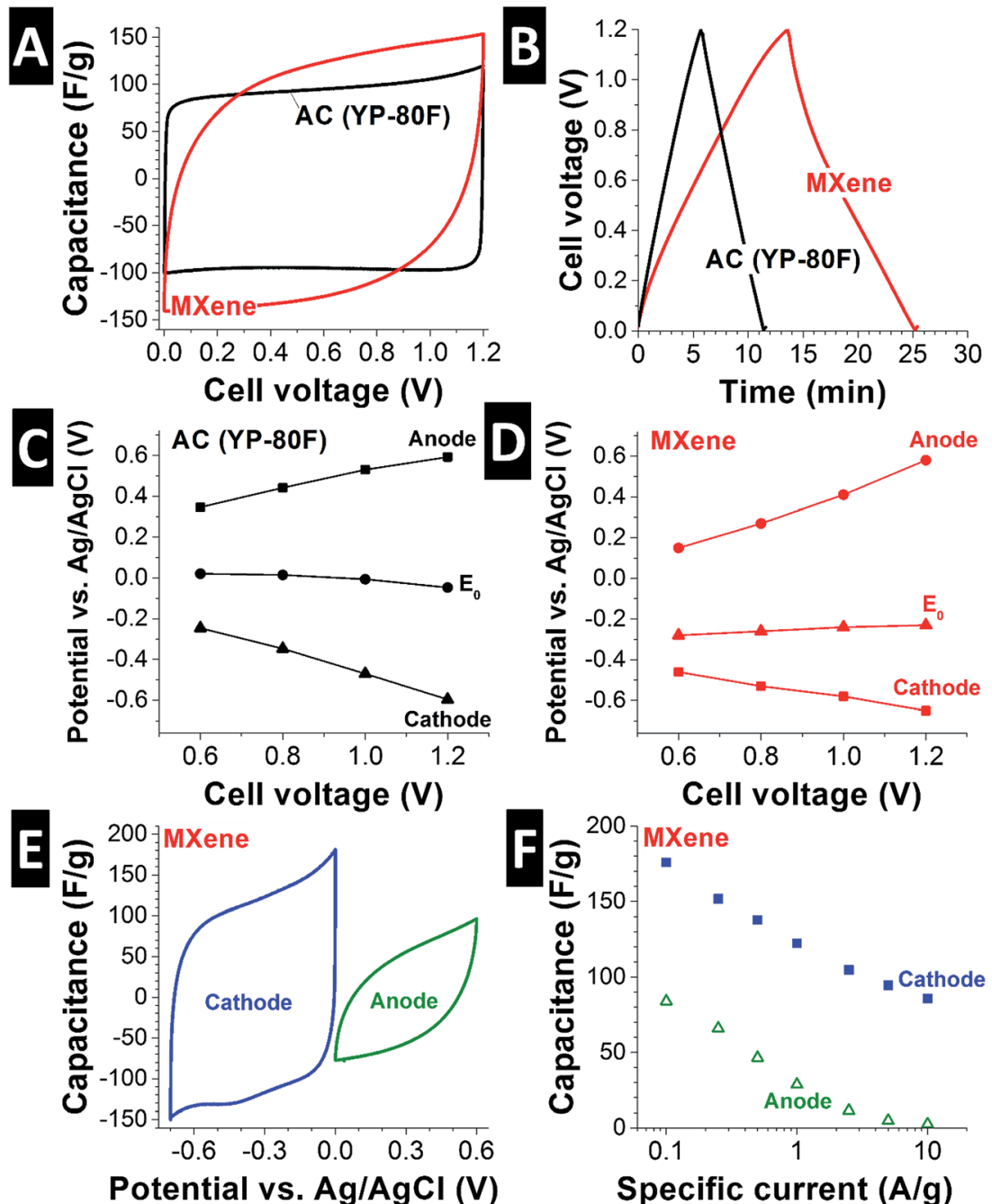

Fig. 2 (A) Cyclic voltammograms at $5 \mathrm{mV} \mathrm{s}^{-1}$ and (B) galvanostatic charge/discharge at $0.1 \mathrm{~A} \mathrm{~g}^{-1}$ of MXene and activated carbon (YP-80F; ref. 26) in $1 \mathrm{M} \mathrm{NaCl}$. (C and D) Electrode potential at different cell voltages for anode and cathode of $\mathrm{MXene}$ and $\mathrm{YP}-80 \mathrm{~F}$ in $1 \mathrm{M} \mathrm{NaCl}$. (E) $\mathrm{Cyclic}$ voltammograms at $5 \mathrm{mV} \mathrm{s}^{-1}$ and (F) galvanostatic charge/discharge power handling for MXene half-cell vs. an oversized YP-80F counter electrode in $1 \mathrm{M} \mathrm{NaCl}$. 
capacitor-like charge/discharge behavior, which manifests in near-linear voltage curves from galvanostatic charge/discharge cycling (Fig. 2C). At a discharge rate of $0.1 \mathrm{~A} \mathrm{~g}^{-1}$, capacitance values of $111 \mathrm{~F} \mathrm{~g}^{-1}$ and $132 \mathrm{~F} \mathrm{~g}^{-1}$ were measured for YP-80F activated carbon and MXene, respectively (Fig. 2B). Considering the low surface area of MXene, the high measured capacitance cannot be due to ions electrosorbed at the fluid/solid interface (like in activated carbon), but instead is attributed to inserted (intercalated) ions between MXene sheets. By this virtue, MXene qualifies not as a double-layer capacitor, but as a pseudocapacitor. ${ }^{22}$

The electrochemical data shown in Fig. 2A and B were derived with a symmetrical two-electrode cell. From galvanostatic charging and discharging at $0.1 \mathrm{~A} \mathrm{~g}^{-1}$ to $1.2 \mathrm{~V}$ cell voltage, a high coulombic efficiency ( $>97 \%$; ratio of discharge $v s$. charge) was calculated. This value indicates a highly reversible charge transfer, for example, accomplished by anion insertion at the positively polarized electrode and cation insertion at the opposite electrode. While most works in the literature have investigated cation intercalation in MXenes, ${ }^{22}$ recent works provide experimental evidence also for anion insertion derived from in situ X-ray diffraction experiments. ${ }^{33}$ By that virtue, MXene is an interesting model material for applying faradaic anion and cation immobilization towards pseudocapacitive desalination.

To further investigate the difference in electrode potential development between activated carbon and MXene electrodes, we added a spectator reference electrode $(\mathrm{Ag} / \mathrm{AgCl})$ to the symmetric two-electrode cells (Fig. 2C and D) in $1 \mathrm{M} \mathrm{NaCl}$. The activated carbon electrode pair showed the behavior expected for a symmetric double-layer capacitor: both electrodes see, by increment and opposite by sign, the same increase in electrode potential as the cell voltage is increased. For example, a cell voltage of $1.0 \mathrm{~V}$ is accomplished by an anode at $+0.5 \mathrm{~V}$ and a cathode at $-0.5 \mathrm{~V}$ vs. $\mathrm{Ag} / \mathrm{AgCl}$ (Fig. 2C). MXene electrodes exhibit a different behavior: compared to activated carbon, the potential of the MXene electrode pair is shifted to negative values $\left(E_{0}\right)$ by ca. $250 \mathrm{mV} v s$. $\mathrm{Ag} / \mathrm{AgCl}$ (Fig. $2 \mathrm{D}$ ). The cell voltage is also differently distributed: a cell voltage of $1.0 \mathrm{~V}$ is accomplished by an anode at $+0.4 \mathrm{~V}$ and a cathode at $-0.6 \mathrm{~V} v s$. $\mathrm{Ag} / \mathrm{AgCl}$. This behavior is in line with the negative static charge of MXenes because of intrinsic $-\mathrm{OH},=\mathrm{O}$ and $-\mathrm{F}$ surface termination. $^{34}$

When performing half-cell experiments vs. an oversized activated carbon counter electrode (YP-80F), we can further investigate the performance of MXene as anode and cathode, that is, during positive or negative polarization. As seen from Fig. 2E, both anodic and cathodic sweeping via cyclic voltammetry $\left(5 \mathrm{mV} \mathrm{s}^{-1}\right)$ yields pseudocapacitive behavior. During positive polarization, a high specific capacitance of $176 \mathrm{~F} \mathrm{~g}^{-1}$ is reached, while negative polarization only provides $84 \mathrm{~F} \mathrm{~g}^{-1}$ (calculated from galvanostatic discharge at $0.1 \mathrm{~A} \mathrm{~g}^{-1}$; see Fig. 2F). Evidently, cation insertion is favored, but the ability of MXene to intercalate ions is not limited to cations as seen from the ability of the MXene anode to demonstrate pseudocapacitive charge/discharge characteristics. Surveying different specific currents (Fig. 2F) and from the shape of the $\mathrm{CV}$ in Fig. 2E, we also see slower anion intercalation and a more rapid loss of capacitance at higher rates.

To ensure stable performance, the MXene electrodes were cycled between 0 and $1.2 \mathrm{~V}$ for 40 times prior CDI experiments in $5 \mathrm{mM} \mathrm{NaCl}$. After this conditioning and wetting process, stable desalination performance was observed with characteristic changes in the effluence salt concentration recorded during potentiostatic cycling (Fig. 3A). The concentration changes compute, based on the averaged data from two separate experiments, to a salt adsorption capacity of $13 \pm 2 \mathrm{mg} \mathrm{g}^{-1}$ at an average salt adsorption rate (ASAR) of $c a .1 \mathrm{mg} \mathrm{g}^{-1} \mathrm{~min}^{-1}$.
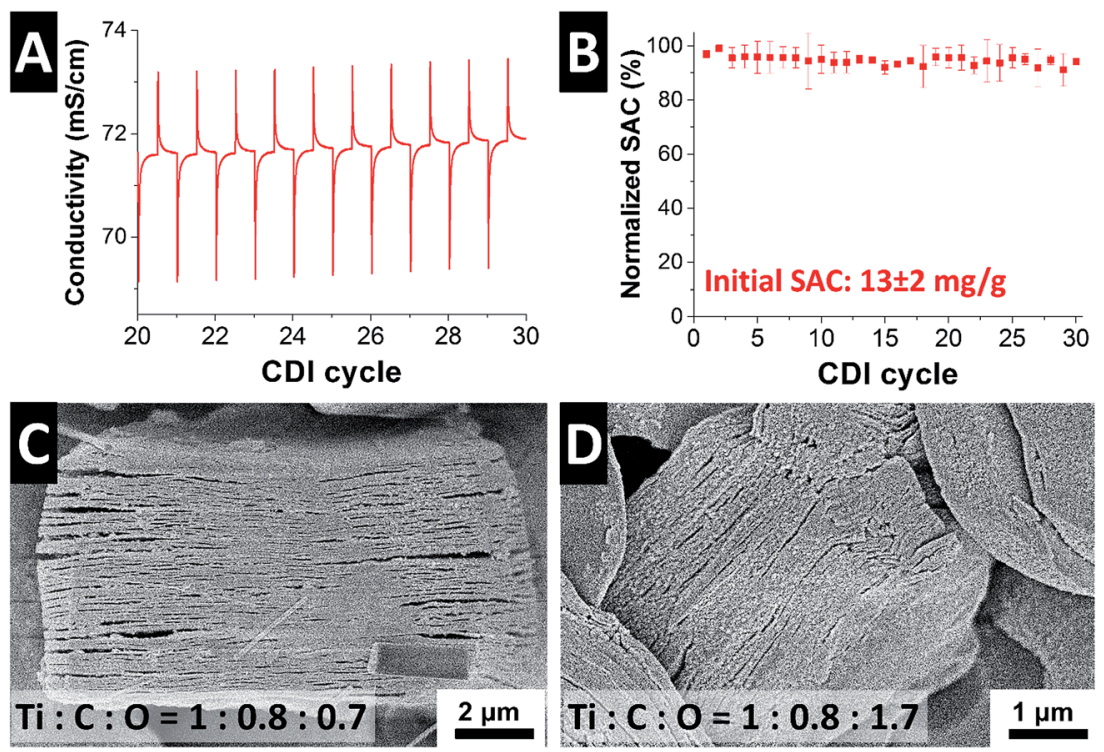

Fig. 3 (A) Conductivity profiles for 10 full cycles in $5 \mathrm{mN} \mathrm{NaCl}$ (deaerated) and (B) corresponding salt removal capacity stability of MXene. Error bars in (B) represent the data for two repeat experiments. Post mortem scanning electron micrographs of $\mathrm{Ti}_{3} \mathrm{C}_{2} \mathrm{MXene}$ after $\mathrm{CDI}$ cycling as (C) negatively and (D) positively polarized electrode. The elemental (molar) ratios noted in (C) and (D) were derived from EDX analysis. 

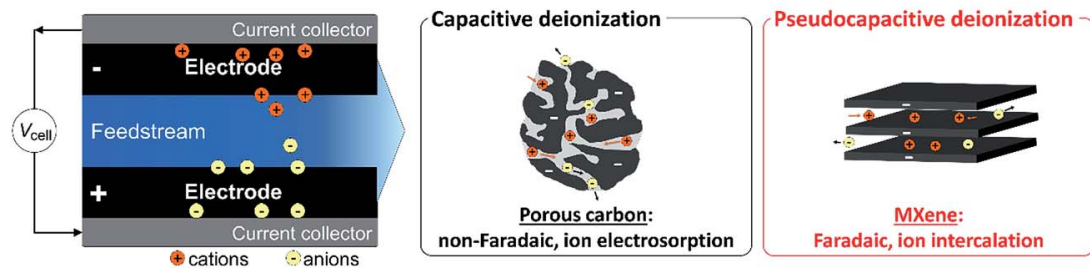

Fig. 4 Concept of electrochemical water desalination. Capacitive deionization accomplishes water desalination by non-faradaic (electrostatic) ion electrosorption at the surface of electrode materials. Pseudocapacitive deionization is accomplished by faradaic ion intercalation between the 2D-sheets of MXene. In both cases, anions and cations are removed.

This value is comparable to YP-80F $\left(9 \mathrm{mg} \mathrm{g}^{-1}\right)$ and MSP-20 $\left(14 \mathrm{mg} \mathrm{g}^{-1}\right)$, but higher than other carbons (like carbon xerogel; $\left.3.3 \mathrm{mg} \mathrm{g}^{-1}\right){ }^{35}$ When normalized to the available surface area, we see that MXene accomplishes a desalination capacity of $1.7 \mathrm{mg}$ $\mathrm{m}^{-2}$, while YP-80F shows an area-normalized SAC of just $4.3 \mu \mathrm{g}$ $\mathrm{m}^{-2}$. This shows that the ion removal for CDI with MXene cannot be accomplished by the outer surface area, but by ion insertion between the MXene sheets (intercalation). In $5 \mathrm{mM}$ de-aerated $\mathrm{NaCl}$ saline solution, the $\mathrm{CDI}$ cell exhibited stable performance over 30 cycles (Fig. 3B).

Initial post mortem analysis of the MXene electrodes shows that the overall "open book" morphology is maintained (Fig. 3C and D). Yet, compared to the initial material (Fig. 1B), the MXene electrodes after positive (Fig. 3C) or negative (Fig. 3D) polarization appear more exfoliated. Importantly, we see an increase of the Ti: C:O molar ratio, from initially $1: 1: 0.5$ (directly after synthesis) to a value of $1: 0.8: 0.7$ for cathode and $1: 0.8: 1.7$ for the anode (post mortem; see also Table 1 ). For ideal $\mathrm{Ti}_{3} \mathrm{C}_{2}$, we would expect a $\mathrm{Ti}: \mathrm{C}$ ratio of $1: 0.7$; thus, the higher initial value of the electrode is because of the presence of excess carbon and small amounts of TiC, as common for MXene. ${ }^{30}$ Oxidation of MXene is well-known ${ }^{36}$ and occurs in the aqueous medium at both electrodes. Triggered by electrochemical oxidation, MXene oxidation occurs to a larger degree at the anode. The reduced relative amount of carbon is a direct result of the carbide oxidation, where carbon is replaced by oxygen in the solid. The combination of repeated ion intercalation/deintercalation and superficial oxidation enhances exfoliation and the morphology of MXene slightly changes. Yet, MXene grains remain overall structural integrity.

\section{Conclusion}

MXene is an interesting novel electrode material for CDI and can be used in a symmetric cell configuration for stable water desalination. The flake structure of the material also enables direct casting onto the porous separator without the need of adding polymer binder. We demonstrated that MXene CDI electrodes accomplished stable performance for 30 cycles with a salt adsorption capacity (SAC) of $13 \pm 2 \mathrm{mg} \mathrm{g}^{-1}(1.2 \mathrm{~V}$ cell voltage in $5 \mathrm{mM} \mathrm{NaCl}$ saline solution). Thus our results show that the MXene SAC is higher than that of many activated carbons and carbon aerogels commonly used for CDI. However, MXene accomplishes adsorption via a completely different ion immobilization mechanism, that is, by ion intercalation instead of double-layer formation, schematically depicted in Fig. 4. That our initial proof-of-concept cell achieves high performance demonstrates that MXene and CDI via intercalation electrodes is a promising direction.

More work is required to better understand the processes of desalination via ion insertion in the MXene layered structure and a more rigorous understanding of the concept of charge efficiency in the context of intercalation materials. Also, enhanced performance may be enabled by use of asymmetric MXene CDI cells and hybrid electrodes. We believe that the field of CDI research will see diversification in the near future beyond carbon by employing pseudocapacitive or battery-like processes for enhanced electrochemical desalination, as already predicted by theoretical work. ${ }^{37}$

\section{Acknowledgements}

The authors thank Prof. Eduard Arzt (INM) for his continuing support. We also acknowledge support of the Prof. Lenz Foundation.

\section{References}

1 M. E. Suss, S. Porada, X. Sun, P. M. Biesheuvel, J. Yoon and V. Presser, Energy Environ. Sci., 2015, 8, 2296-2319.

2 S. Porada, G. Feng, M. E. Suss and V. Presser, RSC Adv., 2016, 6, 5865-5870.

3 S. Porada, L. Borchardt, M. Oschatz, M. Bryjak, J. S. Atchison, K. J. Keesman, S. Kaskel, P. M. Biesheuvel and V. Presser, Energy Environ. Sci., 2013, 6, 3700-3712.

4 X. Xu, L. Pan, Y. Liu, T. Lu, Z. Sun and D. H. C. Chua, Sci. Rep., 2015, 5, 8458.

5 X. Xu, Z. Sun, D. H. C. Chua and L. Pan, Sci. Rep., 2015, 5, 11225.

6 X. Gao, S. Porada, A. Omosebi, K.-L. Liu, P. M. Biesheuvel and J. Landon, Water Res., 2016, 92, 275-282.

7 X. Gao, A. Omosebi, J. Landon and K. L. Liu, Energy Environ. Sci., 2015, 8, 897-909.

8 B. P. Bastakoti, H. Oveisi, C.-C. Hu, K. C. W. Wu, N. Suzuki, K. Takai, Y. Kamachi, M. Imura and Y. Yamauchi, Eur. J. Inorg. Chem., 2013, 1109-1112.

9 B. P. Bastakoti, Y. Kamachi, H.-S. Huang, L.-C. Chen, K. C. W. Wu and Y. Yamauchi, Eur. J. Inorg. Chem., 2013, 39-43. 
10 J. Lee, S. Kim, C. Kim and J. Yoon, Energy Environ. Sci., 2014, 7, 3683-3689.

11 S. Dutta, S.-Y. Huang, C. Chen, J. E. Chen, Z. A. Alothman, Y. Yamauchi, C.-H. Hou and K. C. W. Wu, ACS Sustainable Chem. Eng., 2016, 4, 1885-1893.

12 N.-L. Liu, S. Dutta, R. R. Salunkhe, T. Ahamad, S. M. Alshehri, Y. Yamauchi, C.-H. Hou and K. C. W. Wu, Sci. Rep., 2016, 6, 28847.

13 A. K. Geim and K. S. Novoselov, Nat. Mater., 2007, 6, 183-191.

14 A. Gupta, T. Sakthivel and S. Seal, Prog. Mater. Sci., 2015, 73, 44-126.

15 M. Naguib, V. N. Mochalin, M. W. Barsoum and Y. Gogotsi, Adv. Mater., 2014, 26, 992-1005.

16 M. Naguib, M. Kortoglu, V. Presser, J. Lu, L. Hultman, J. Niu, M. Heon, Y. Gogotsi and M. W. Barsoum, Adv. Mater., 2011, 23, 4248-4253.

17 M. Naguib and Y. Gogotsi, Acc. Chem. Res., 2014, 48, 128-135. 18 U.S. Department of Energy, Carbon Sequestration Technology and Program Plan, 2007.

19 M. R. Lukatskaya, O. Mashtalir, C. E. Ren, Y. Dall'Agnese, P. Rozier, P. L. Taberna, M. Naguib, P. Simon, M. W. Barsoum and Y. Gogotsi, Science, 2013, 341, 15021505.

20 M. R. Lukatskaya, J. Halim, B. Dyatkin, M. Naguib, Y. S. Buranova, M. W. Barsoum and Y. Gogotsi, Angew. Chem., Int. Ed., 2014, 53, 4877-4880.

21 F. Shahzad, M. Alhabeb, C. B. Hatter, B. Anasori, S. Man Hong, C. M. Koo and Y. Gogotsi, Science, 2016, 353, 1137.

22 M. D. Levi, M. R. Lukatskaya, S. Sigalov, M. Beidaghi, N. Shpigel, L. Daikhin, D. Aurbach, M. W. Barsoum and Y. Gogotsi, Adv. Energy Mater., 2015, 5, 1400815.

23 J. Come, J. M. Black, M. R. Lukatskaya, M. Naguib, M. Beidaghi, A. J. Rondinone, S. V. Kalinin, D. J. Wesolowski, Y. Gogotsi and N. Balke, Nano Energy, 2015, 17, 27-35.
24 Z. Lin, D. Barbara, P.-L. Taberna, K. L. Van Aken, B. Anasori, Y. Gogotsi and P. Simon, J. Power Sources, 2016, 326, 575-579.

25 M. Hu, Z. Li, H. Zhang, T. Hu, C. Zhang, Z. Wu and X. Wang, Chem. Commun., 2015, 51, 13531-13533.

26 M. Aslan, M. Zeiger, N. Jäckel, I. Grobelsek, D. Weingarth and V. Presser, J. Phys.: Condens. Matter, 2016, 28, 114003.

27 S. Brunauer, P. H. Emmett and E. Teller, J. Am. Chem. Soc., 1938, 60, 309-319.

28 N. Jäckel, M. Rodner, A. Schreiber, J. Jeongwook, M. Zeiger, M. Aslan, D. Weingarth and V. Presser, J. Power Sources, 2016, 326, 660-671.

29 S. Porada, R. Zhao, A. van der Wal, V. Presser and P. M. Biesheuvel, Prog. Mater. Sci., 2013, 58, 1388-1442.

30 M. Naguib, O. Mashtalir, J. Carle, V. Presser, J. Lu, L. Hultman, Y. Gogotsi and M. W. Barsoum, ACS Nano, 2012, 6, 1322-1331.

31 J. Halim, K. M. Cook, M. Naguib, P. Eklund, Y. Gogotsi, J. Rosen and M. W. Barsoum, Appl. Surf. Sci., 2016, 362, 406-417.

32 J. Halim, S. Kota, M. R. Lukatskaya, M. Naguib, M.-Q. Zhao, E. J. Moon, J. Pitock, J. Nanda, S. J. May, Y. Gogotsi and M. W. Barsoum, Adv. Funct. Mater., 2016, 26, 3118-3127.

33 Z. Lin, P. Rozier, B. Duployer, P.-L. Taberna, B. Anasori, Y. Gogotsi and P. Simon, Electrochem. Commun., 2016, 72, 50-53.

34 M. A. Hope, A. C. Forse, K. J. Griffith, M. R. Lukatskaya, M. Ghidiu, Y. Gogotsi and C. P. Grey, Phys. Chem. Chem. Phys., 2016, 18, 5099-5102.

35 J. Landon, X. Gao, B. Kulengowski, J. K. Neathery and K. Liu, J. Electrochem. Soc., 2012, 159, A1861-A1866.

36 M. Naguib, O. Mashtalir, M. R. Lukatskaya, B. Dyatkin, C. Zhang, V. Presser, Y. Gogotsi and M. W. Barsoum, Chem. Commun., 2014, 50, 7420-7423.

37 K. C. Smith and R. Dmello, J. Electrochem. Soc., 2016, 163, A530-A539. 